\title{
A recursive methodology for modelling multi-stranded wires with multilevel helix structure
}

\author{
Emilio Plumed $^{1 *}$, Ignacio Lope ${ }^{2,3}$, Claudio Carretero ${ }^{2}$, Jesús Acero $^{1}$ \\ ${ }^{1}$ Departamento de Ingeniería Electrónica y Comunicaciones, Universidad de Zaragoza, \\ 50018 Zaragoza, Spain. \\ ${ }^{2}$ Departamento de Física Aplicada, Universidad de Zaragoza, 50009 Zaragoza, Spain. \\ ${ }^{3} \mathrm{~B} / \mathrm{S} / \mathrm{H} / \mathrm{Home}$ Appliances, 50016 Zaragoza, Spain.
}

\begin{abstract}
Multi-stranded litz wires are commonly used in magnetic devices for power electronics applications at medium-high frequency range, from several $\mathrm{kHz}$ up to hundreds of kHz. For these applications, litz-wire structure favours the uniformity of driven current in the cross-sectional area of conductors, alleviating ac losses (skin and proximity effects) and improving the global efficiency of the application. These features are achieved by means of a special cable arrangement consisting of many isolated fine copper strands twisted together according to the manufacturing process. Often, the manufacturing process involves several twisting steps where bundles of moderate number of strands are successively twisted resulting in intricate cable structures. We present a mathematical description of the trajectories of copper strands with the purpose of obtaining the cable losses by means of Finite Element Analysis (FEA) simulation tools. Moreover, a nomenclature for this multilevel structures is also proposed. Parameters as the number of twisting steps, number of strands, strand diameter or pitch length, are included in this representation, allowing to compare the performance of different manufacturing solutions.

Keywords: Frenet-Serret frame; electromagnetic analysis; eddy

currents; conductors; proximity effects; finite element analysis
\end{abstract}

Preprint submitted to Applied Mathematical Modelling

February 6, 2020 


\section{Introduction}

Litz wires are becoming the cable of choice in many power electronic fields because they mitigate the severe eddy-current losses of conductors which occurs in energy conversion applications at medium-high frequency regimes. Wireless Inductive Power Transfer (IPT) Systems [1], domestic induction heating [2] and, in general, magnetic design [3] are some examples of areas of interest. Accordingly, aspects as optimization, loss modelling, and simulation are of current interest. Moreover, considering that, in general, the finer the strands, the more efficiency is achieved and the higher the cost is, it also exists a practical interest in balancing performance against cost.

Ac loss reduction is achieved when a thick cable is divided in several preferably isolated fine strands. The number of strands of a cable is commonly denoted as $n_{s}$. Moreover, if some transposition pattern is applied to strands, which favours the geometrical equivalence of strands, additional loss reductions are achieved. Litz wires are wound by recursively twisting bundles of strands together in multiple levels. Usually, the innermost level has a moderate number of thin strands, which are twisted such that the trajectory of each strand corresponds to a helix. The twisting is repeated recursively on the bundles to form bundles of higher level, as is shown in Fig. 1p.

Magnetic design with litz wire requires an estimation of ac losses with respect to the cable parameters: number of strands, structure of the cable, and diameter of strands. Existing loss models essentially rely on three assumptions: equivalence of strands, orthogonality of ac loss mechanisms (skin and proximity effects), and widely spaced isolated and infinite strands. The preceding premises implicitly assume that cable structure is perfect and therefore current is equally shared in strands, which minimizes the Joule's effect losses. Accordingly, ac losses of litz wires are usually computed as the losses of a strand multiplied by the number of strands.

Ac losses in a strand are calculated as the sum of copper losses due to skin and proximity effects, which in its turn can be obtained as exact solutions 


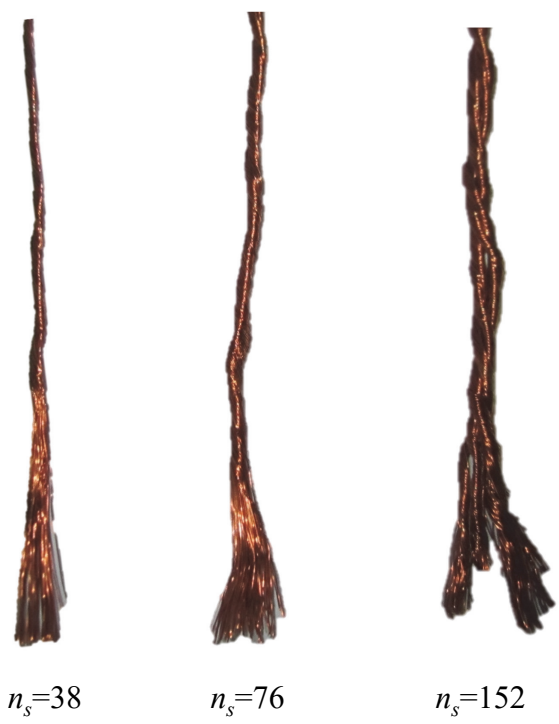

Figure 1: Litz wires with different number of strands and structure: $n_{s}=38$ single bundle, $n_{s}=76$ in two bundles of previous wire, $n_{s}=156$ in two bundles of previous wire.

of Maxwell's equations for round geometry by applying the third premise as boundary condition [4]. These ideas are in the heart of existing loss models [5, 6, 17, 8, 9, 10, 11, 12. Similarly, proposed optimization procedures for designs with litz wires are also based on these assumptions [13, 14].

Several loss analyses of litz wire arrangements can be found in the literature, both analytical and numerical approaches. An example of an analytical approach is proposed in [15, where, apart from the loss analysis, a nomenclature is also proposed for the case of multi-level arrangements.

Considering numerical analyses, 2-D FEA-based models have been mainly proposed, where axial symmetry with respect to the cable axis is assumed [16, 17]. In these approaches, strands placed in the bundle at the same radial position are indistinguishable and, therefore twisting effects as the cancellation of external flux are not captured and often are introduced by means of extra boundary conditions. Comparatively, 3-D FEA approaches are much less frequent and are restricted to the case of one bundle of several strands [18, 19]. In these approaches, strand trajectories are defined by means of helical curves and, therefore, complex multilevel bundle structures are not considered. 
Apart from FEA tools, other numerical methods as the Partial Element Equivalent Circuit (PEEC) have been used to analyze litz wires with several bundle levels. This method transforms the system under electromagnetic study into an equivalent circuit, by subdividing the conductive regions in filaments and assigning self and mutual inductances between them [20]. Other works combine FEA and PEEC methods in order to take into account the effect of media which are usually placed near the conductors, for example magnetic cores 21]. Approaches using integral equations have been also proposed [22].

Despite the mentioned models having achieved accurate predictions in most cases, some aspects of litz-wire loss modelling are still pending of a satisfactory approach, specially those aspects related with the underlying structure of the cable. Details of construction of the wire, i.e. complex structures which can comprise several bundle levels (as it is shown in Fig. 1) can become relevant. These reasons are often mentioned when discrepancies between theory and measurements are found. Structure of litz wire and wire rope are, in general terms, identical. The basic element of both is a single thin metallic wire. However, in the field of the litz wire the individual solid wire are previously insulated and is properly called magnet wire. In the past, several descriptions of the structure of wire rope have been proposed [23, 24, 25], mainly with the purpose of estimating the mechanical stresses of the wire by means of finite element simulations.

In a previous work, [26, a mathematical description of litz-wire arrangements was presented with the purpose of obtaining strand resistances and current densities by means of 3-D FEA simulations. The analysed case consisted of a single structure with two different cable pitch lengths, whose effect in skin and proximity losses was established. In this present work, the mathematical description has been expanded by incorporating elements from wire rope descriptions, particularly the collision avoidance methods. A rigorous method and a simplified method have been tested for their solvability, accuracy and applicability to the current problem. Additionally, the simulated cases seek to determine the dependencies of the ac losses with respect to different constructive parameters (as the number strands per bundle and the insulation between 


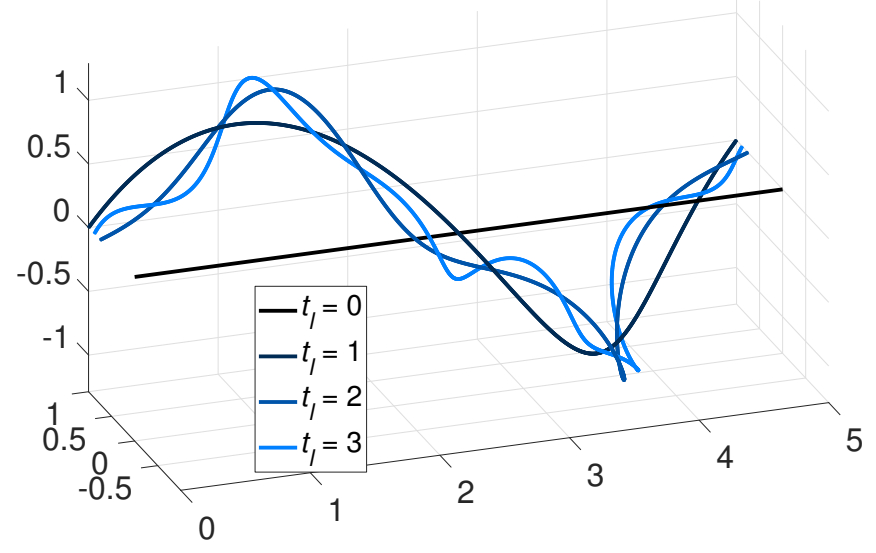

Figure 2: Generation of helix bundle trajectories.

strands). These dependencies show some manufacturing guidelines which can help to reduce cable losses.

\section{Mathematical description}

Electromagnetic analysis of a litz wires under ac-excitation conditions can be carried out by means of 3-D FEA simulations. However, these 3-D simulations present some challenges, mainly derived from the fact that litz wire is a multiscale structure where extremely different orders of magnitude can appear in the same system under study. For example, in real applications it is usual to find strands with diameters of microns whose total lengths can be up to several meters. Moreover, it is also very common the use of wires with hundreds or thousands of isolated strands, manufactured in several bundling steps, whose analysis capturing the geometrical details is also challenging. Actually, realistic simulations of real applications with litz wires is almost unapproachable even with advanced computing facilities.

\subsection{Nomenclature and definitions}

Equivalent elements of wire rope and litz wire are typically described with different terms, so in order to avoid confusion, next are the terms that will be used throughout the paper. Each individual length of conductive metal is 


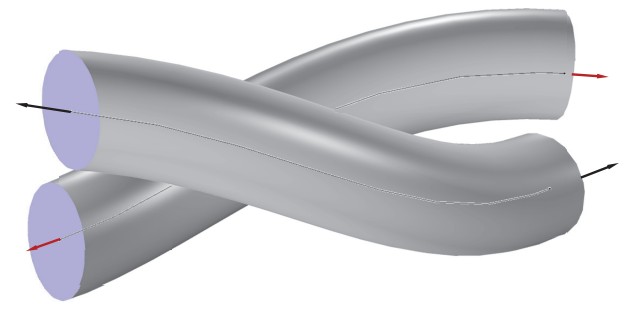

Figure 3: Generation of strand domains.

called a strand. Several strands can be twisted together to form bundles. Several bundles can in turn compose more complex bundles. The grouping of all bundles is called the (litz) wire.

Construction details of a litz wire can include many factors and, for this reason, the following basic assumptions are adopted in this work:

- Only round strands are considered.

- Strands have no electrical contact and the insulation material is the air.

- Smooth trajectories of both strands and bundles, which can be described in the Frenet-Serret frame, are considered.

- A bundling operation involves identical sub-bundles.

In the FEA tool, in this case COMSOL, the domain of every strand can be generated by extruding a circle along a given trajectory as it is schematically shown in Fig. 1). Therefore, it is necessary to describe mathematically the trajectory of every strand for simulating the cable. For this purpose, it is convenient to define some parameters in order to describe complex wire structures. Moreover, it is also of interest to define a nomenclature to properly denote structures made up of several bundles, which can in turn hold even smaller bundles.

In this work, the term bundle refers to the basic entity. The external radius of a bundle is denoted as $r_{b}$. Considering that a bundle, as a result of a twisting process, can include several concentric layers of strands, in general, it is considered that a bundle is placed in the $n$ layer of a more general bundle and has 
a position radius $r_{\text {pos, } n}$ with respect to the center of the bundle. Therefore, $r_{b}$ can be calculated from the position of the outermost sub-bundle and its external radius: $r_{b}=r_{\text {pos }, n_{\max }}+r_{b, n_{\max }}$. The number of layers of a bundle is $n_{b}$. Other parameters of interest for describing a bundle are the bundle level $b_{l}$ and the twisting level $t_{l}$, which correspond to the number of bundling and twisting operations, respectively, conducted on a bundle in the manufacturing process of the cable.

Strands are placed in the deepest bundle level and, for convenience, they are considered bundles as well. A single straight strand corresponds to a bundle with $n_{b}=0, b_{l}=0$, and $t_{l}=0$. Similarly, central bundles (or central strands) are placed in the layer $n=0$ of a bundle and have one twisting level less than the rest of entities of the bundle.

According to the previous definitions, a bundle can be associated to an array $\mathbf{N}_{l}$ of $b_{l}$ elements, where $b_{l}$ is its bundle level. The $i^{\text {th }}$ element corresponds to the $n^{\text {th }}$ layer of the $i^{\text {th }}$ bundle level where the bundle is placed. According to this definition, parameters $b_{l}$ and $t_{l}$ of a bundle can be obtained from $\mathbf{N}_{l}$. In particular $b_{l}$ is equal to the dimension of $\mathbf{N}_{l}$ and $t_{l}$ corresponds to the number of elements of $\mathbf{N}_{l}$ higher than zero. Fig. 4 shows some examples of values of $\mathbf{N}_{l}$ for different bundles for a cable of $14 \times 7 \times 3$ entities.

Regarding trajectories, it is considered that bundles describe circles along a path. In particular, if the path is simply a straight line, as it is schematically shown in Fig. 2, the trajectory corresponds to a helix. Small bundles twisted together also describe helical trajectories, as it is schematically shown in Fig. 2 . In general, the trajectory of the $\mathbf{N}_{l}$ bundle can be mathematically described by considering that it successively describes $t_{l}$ helices with respect to $b_{l}$ trajectories.

\subsection{Mathematical description of bundle trajectories}

In general, bundles describe helices around a core according to its position radius $r_{\mathrm{pos}, n}$, therefore the case of straight bundles corresponds to $r_{\mathrm{pos}, n=0}=0$. If the core is a straight line, the bundle trajectory for a given position radius 


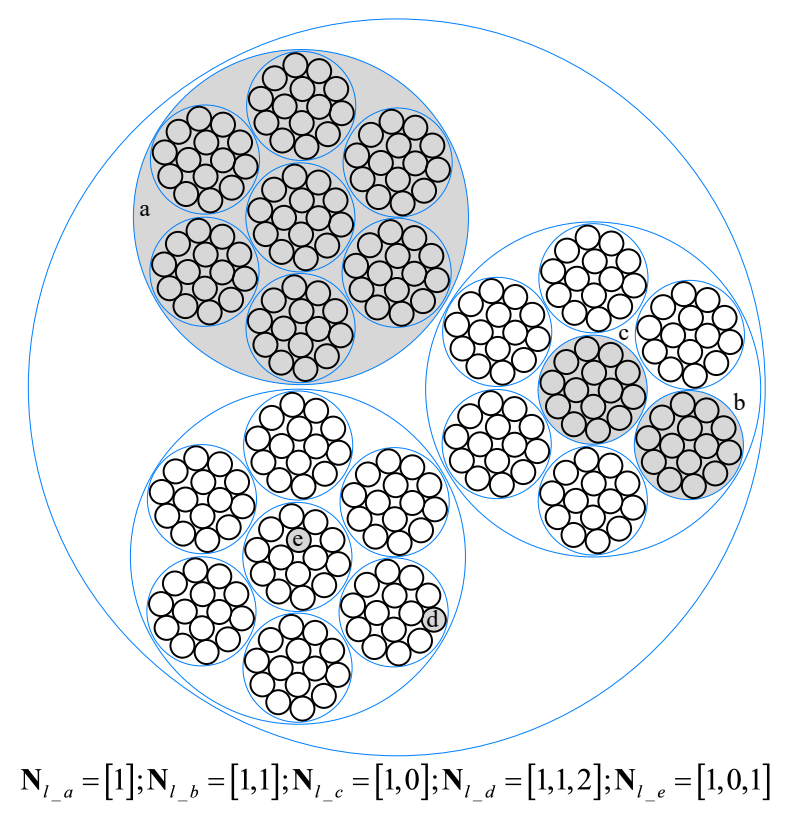

Figure 4: Examples of array $\mathbf{N}_{l}$ for different bundles.

$r_{\mathrm{pos}, n}$ corresponds to a helix whose parameterized equation is:

$$
\left\{\begin{array}{l}
x=\lambda \varphi \\
y=r_{\mathrm{pos}, n} \cdot \cos \varphi \\
z=r_{\mathrm{pos}, n} \cdot \sin \varphi
\end{array}\right.
$$

where $\varphi$ is the curve parameter that ranges from 0 to $2 \pi$ for a single helix turn, $\lambda$ is the angular pitch, $\lambda=\lambda / 2 \pi$, where $\lambda$ is the pitch in a similar relation between linear and angular wavelength.

In order to describe the trajectory of bundles, it is convenient to adopt the Frenet-Serret frame [27] in which smooth curves are described on the basis of three orthonormal unitary vectors (i.e. directional vectors) $\hat{\mathbf{t}}, \hat{\mathbf{n}}, \hat{\mathbf{b}}$ called tangent, normal and binormal, respectively. Moreover, as it has been mentioned, the manufacturing of a litz wire can be assimilated to a recursive process and the trajectory and position of a given bundle for a twist level can be described on the basis of the core trajectory of the previous twist level:

$$
\mathbf{r}_{t_{l}}=\mathbf{r}_{t_{l}-1}+r_{\mathrm{pos}, n} \cos \left(p r_{n} \cdot \varphi\right) \cdot \hat{\mathbf{n}}_{t_{l}-1}+_{\mathrm{pos}, n} \sin \left(p r_{n} \cdot \varphi\right) \cdot \hat{\mathbf{b}}_{t_{l}-1},
$$

where $\mathbf{r}_{t_{l}}$ is the position vector of the twist level $t_{l}, p r_{n}$ is the ratio of the $n^{\text {th }}$ layer pitch of the $t_{l}^{\text {th }}$ twist level with respect to the global wire pitch and $\hat{\mathbf{n}}_{t_{l}-1}$ 
and $\hat{\mathbf{b}}_{t_{l}-1}$ are the normalized perpendicular vectors of the twist level $t_{l}-1$. The parameter $p r_{n}$ takes into account that different twist levels can have different pitches. In order for the wire to be periodic in $2 \pi$ intervals of $\varphi$, all $p r_{n}$ values have to be non-zero integers. Negative values change the lay of the bundle.

The tangent and normal vectors of each twist level can be obtained as follows:

$$
\begin{aligned}
\overline{\mathbf{t}}_{t_{l}} & =\frac{\partial x_{t_{l}}}{\partial \varphi} \hat{\mathbf{x}}+\frac{\partial y_{t_{l}}}{\partial \varphi} \hat{\mathbf{y}}+\frac{\partial z_{t_{l}}}{\partial \varphi} \hat{\mathbf{z}} \\
\overline{\mathbf{n}}_{t_{l}} & =\frac{\partial\left(t_{t_{l}}\right)_{x}}{\partial \varphi} \hat{\mathbf{x}}+\frac{\partial\left(t_{t_{l}}\right)_{y}}{\partial \varphi} \hat{\mathbf{y}}+\frac{\partial\left(t_{t_{l}}\right)_{z}}{\partial \varphi} \hat{\mathbf{z}} \\
\hat{\mathbf{t}}_{t_{l}} & =\frac{\overline{\mathbf{t}}_{t_{l}}}{\left|\overline{\mathbf{t}}_{t_{l}}\right|}, \\
\hat{\mathbf{n}}_{t_{l}} & =\frac{\overline{\mathbf{n}}_{t_{l}}}{\left|\overline{\mathbf{n}}_{t_{l}}\right|} \\
\hat{\mathbf{b}}_{t_{l}} & =\hat{\mathbf{t}}_{t_{l}} \times \hat{\mathbf{n}}_{t_{l}} .
\end{aligned}
$$

Apart from calculating the position vector, it is also convenient to obtain the rest of directional vectors because they are required to calculate the current density and losses in conductors. Considering the twist level $t_{l}=1$, the directional vectors are:

$$
\begin{aligned}
& \hat{\mathbf{t}}_{1}=\frac{\lambda}{\sqrt{\lambda^{2}+r_{\mathrm{pos}, 1}^{2}}} \hat{\mathbf{x}}-\frac{r_{\mathrm{pos}, 1} \cdot \sin \left(p r_{1} \cdot \varphi\right)}{\sqrt{\lambda^{2}+r_{\mathrm{pos}, 1}^{2}}} \hat{\mathbf{y}}+\frac{r_{\mathrm{pos}, 1} \cdot \cos \left(p r_{1} \cdot \varphi\right)}{\sqrt{\lambda^{2}+r_{\mathrm{pos}, 1}^{2}}} \hat{\mathbf{z}}, \\
& \hat{\mathbf{n}}_{1}=-\cos \left(p r_{1} \cdot \varphi\right) \hat{\mathbf{y}}+\sin \left(p r_{1} \cdot \varphi\right) \hat{\mathbf{z}}, \\
& \hat{\mathbf{b}}_{1}=\frac{r_{\mathrm{pos}, 1}}{\sqrt{\lambda^{2}+r_{\mathrm{pos}, 1}^{2}}} \hat{\mathbf{x}}+\frac{\lambda \cdot \sin \left(p r_{1} \cdot \varphi\right)}{\sqrt{\lambda^{2}+r_{\mathrm{pos}, 1}^{2}}} \hat{\mathbf{y}}-\frac{\lambda \cdot \cos \left(p r_{1} \cdot \varphi\right)}{\sqrt{\lambda^{2}+r_{\mathrm{pos}, 1}^{2}}} \hat{\mathbf{z}} .
\end{aligned}
$$

The position vector corresponding to the twist level $t_{l}=2$ can be obtained using (2): 


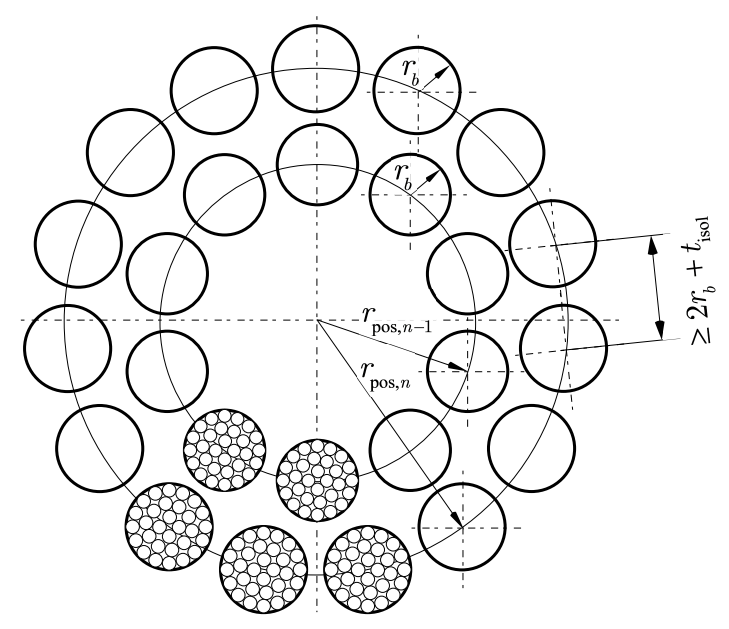

Figure 5: Position radii of two consecutive layers of a bundle.

$$
\begin{aligned}
x_{2} & =\lambda \cdot p r_{1} \cdot \varphi+r_{\mathrm{pos}, 2} \frac{r_{\mathrm{pos}, 1}}{\sqrt{\lambda^{2}+r_{\mathrm{pos}, 1}^{2}}} \sin \left(p r_{2} \cdot \varphi\right) \\
y_{2} & =r_{\mathrm{pos}, 1} \cdot \cos \left(p r_{1} \cdot \varphi\right)-r_{\mathrm{pos}, 2} \cdot \cos \left(p r_{1} \cdot \varphi\right) \cdot \cos \left(p r_{2} \cdot \varphi\right)+ \\
& +r_{\mathrm{pos}, 2} \frac{\lambda \cdot \sin \left(p r_{1} \cdot \varphi\right)}{\sqrt{\lambda^{2}+r_{\mathrm{pos}, 1}^{2}}} \sin \left(p r_{2} \cdot \varphi\right) \\
z_{2} & =r_{\mathrm{pos}, 1} \cdot \sin \left(p r_{1} \cdot \varphi\right)-r_{\mathrm{pos}, 2} \cdot \sin \left(p r_{1} \cdot \varphi\right) \cdot \cos \left(p r_{2} \cdot \varphi\right)- \\
& -r_{\mathrm{pos}, 2} \frac{\lambda \cdot \cos \left(p r_{1} \cdot \varphi\right)}{\sqrt{\lambda^{2}+r_{\mathrm{pos}, 1}^{2}}} \cdot \sin \left(p r_{2} \cdot \varphi\right)
\end{aligned}
$$

The last expressions can be used for obtaining the directional vectors $\hat{\mathbf{t}}_{2}, \hat{\mathbf{n}}_{2}$, and $\hat{\mathbf{b}}_{2}$ by means of (3)-77). Moreover, analytical expressions for position and directional vectors of bundles with $t_{l}=3$ have been also obtained for the simulations presented in section 3. In order to implement these expressions, the position radius of bundles is required.

\subsection{Collision detection methods}

Bundles can be built outwards starting from their core, successively adding the required sub-bundle layers. Therefore, in order to avoid collision, $r_{\mathrm{pos}, n}$ has 
to satisfy two conditions:

- The sub-bundle position radius $r_{\mathrm{pos}, n}$ should be greater than the sum of the sub-bundle's external radius, $r_{b, n}$, and the external radius of the previous layer, $r_{\mathrm{pos}, n-1}+r_{b, n-1}$.

- The bundle position radius should be big enough to place all bundles in the layer, assuming a regular distribution as shown in Fig. 5

Considering bundles composed of identical sub-bundles of radius $r_{b}$, the first condition can be easily expressed:

$$
r_{\text {pos }, n} \geq 2 \cdot r_{b}+t_{\text {isol }}+r_{\text {pos }, n-1}
$$

where $r_{\mathrm{pos}, n-1}$ is the position radius of a bundle placed in the $(n-1)$ layer, $t_{\mathrm{ins}}$ is the insulation distance between bundles and $b_{n}$ is the number of bundles of the layer. The minimum insulation distance corresponds the insulation thickness of strands, which is a standardised value dependent of the insulation grade.

There are several approaches to avoid overlapping between bundles in the same layer. The most rigourous seek to determine the minimum distance between bundles along their entire length [24, 25]. Others simplify the threedimensional problem to a two-dimensional cross section, assuming that the resulting bundle sections will have the shape of ellipses [23. In the case of litz wire, as opposed to cable rope, the bundles and strands do not need to be in physical contact, and in fact they are separated by an insulating layer to avoid electrical contact. A small error in the positioning radius will mean a smaller gap between bundles instead of physical overlap. Therefore, absolutely exact positioning is not strictly required. Nevertheless, both kinds of approaches were tested to be able to compare results.

In the case of litz wires, the bundle's external radius is an input parameter, as it can be calculated incrementally from the bundle composition down to the strand radius. The wire's pitch, $\lambda$, can be defined either directly, or indirectly with the helix angle, $\beta$, through the relation $\lambda=2 \pi r_{\mathrm{pos}, \mathrm{n}} \tan \beta$. Therefore, the 
collision detection methods need to have bundle external radius and pitch as input parameters and minimum bundle position radius as output.

\subsubsection{Three dimensional method}

The three-dimensional method expresses the trajectory of two consecutive bundles (A and B, for example) in the same layer in terms of their curve parameter, the angle $\varphi=\varphi_{A}$, their difference between curve angle, $\Delta \varphi=\varphi_{B}-\varphi_{A}$, and their in-layer angular separation $\theta$, given by the number of bundles in the layer, $\theta=2 \pi / b_{n}$. The segment with minimum distance between $\mathrm{A}$ and $\mathrm{B}$ for each value of $\varphi$ is perpendicular to both trajectories, so this segment's length is simply the sum of the bundles' radius and gap between them. Since the position vector of the bundles are periodic with period $2 \pi$, the minimum distance can be found in any period length interval of $\varphi$. Consequently, the absolute limits of $\Delta \varphi$ are $[-2 \pi, 2 \pi]$ although the minimum distance is expected to be found in values closer to zero.

$$
\begin{aligned}
& \overrightarrow{\mathbf{A B}}=\mathbf{p}_{B}\left(\varphi, \Delta \varphi, \theta, r_{\mathrm{pos}, \mathrm{b}}\right)-\mathbf{p}_{A}\left(\varphi, r_{\mathrm{pos}, \mathrm{b}}\right) \\
& \min \left(\left\|\overrightarrow{\mathbf{A B}}\left(\varphi, \Delta \varphi, \theta, r_{\mathrm{pos}, \mathrm{b}}\right)\right\|\right)=2 r_{b}+t_{\mathrm{ins}}
\end{aligned}
$$

The equation can be transformed to output the bundle position radius:

$$
r_{\mathrm{pos}, \mathrm{b}, \min }=\max \left(r_{\mathrm{pos}, \mathrm{b}}\left(\varphi, \Delta \varphi, \theta, r_{b}, t_{\mathrm{ins}}\right)\right)
$$

Parameters $\theta, r_{b}$ and $t_{\text {ins }}$ are known beforehand, so the function has to be maximized for inputs $\varphi$ and $\Delta \varphi$.

The implementation for a bundle of $t_{l}=1$ is rather straightforward. The vector $\overrightarrow{\mathbf{A B}}_{1}$ can be defined as:

$$
\overrightarrow{\mathbf{A B}_{1}}=\left(\begin{array}{c}
\lambda \Delta \varphi \\
r_{\mathrm{pos}, \mathrm{b}}(\cos (\varphi+\Delta \varphi+\theta)-\cos \varphi) \\
r_{\mathrm{pos}, \mathrm{b}}(\sin (\varphi+\Delta \varphi+\theta)-\sin \varphi)
\end{array}\right) .
$$

Moreover, its squared norm is:

$$
\left\|\overrightarrow{\mathbf{A B}}_{1}\right\|^{2}=\lambda^{2} \Delta \varphi^{2}+2 r_{\text {pos }, \mathrm{b}}^{2}(1-\cos (\Delta \varphi+\theta)) .
$$


Table 1: Parameters used for the convexity tests

\begin{tabular}{cccccccc}
$t_{l}$ & $才$ & $r_{b}$ & $b_{n}$ & $p r_{2}$ & $p r_{3}$ & $r_{\mathrm{pos}, \mathrm{b}, 1}$ & $r_{\mathrm{pos}, \mathrm{b}, 2}$ \\
\hline 1 & 1 & 0.1 & 5 & - & - & - & - \\
2 & 1 & 0.1 & 5 & 2 & - & 1 & - \\
3 & 1 & 0.1 & 5 & 3 & 2 & 1 & 0.5
\end{tabular}

Helices of $t_{l}=1$ have a very regular trajectory, so the distance between two of the same $r_{\text {pos,b }}$ and $\beta$ is only dependent on the difference of position and not its absolute value. Isolating $r_{\mathrm{pos}, \mathrm{b}}$ :

$$
r_{\mathrm{pos}, \mathrm{b}, \min }=\max \left(\sqrt{\frac{\left(2 r_{b}+t_{\mathrm{ins}}\right)^{2}-\lambda^{2} \Delta \varphi^{2}}{2(1-\cos (\Delta \varphi+\theta))}}\right)
$$

As stated earlier, for simple helices the maximization function has only one input parameter, which will not be the case for higher levels where the axial tangent component is not constant. Although this is the simplest position radius expression, it is clearly non linear, and higher level expressions are even more intricate. Since analytical methods are not feasible, numerical ones are the only option. Fortunately, there are myriad numeric methods that can be used to maximize bounded, multiple input, non linear functions.

In this case, since Matlab's symbolic toolbox was already used to obtain the expressions for the position, tangent, normal and binormal vectors, it is also applied to deduce the expressions of $r_{\mathrm{pos}, \mathrm{b}, \mathrm{min}}$ for each $t_{l}$ and eventually maximize them for each geometry case. The Matlab function used was fmincon [28], which minimizes non linear functions with any number of input arguments, user input constraints and bounds on the inputs. The default algorithm uses an interior point method where in each iteration, it attempts a direct, linear approximation step. If the projected Hessian for said step is not positive definite, the algorithm performs a conjugate gradient step. In order to solve the maximization problem with a minimization algorithm, the input function is $-r_{\mathrm{pos}, \mathrm{b}}$.

Most optimization algorithms require a convex function. A scalar function's convexity can be proven if its Hessian is positive definite (i.e. all eigenvalues are 


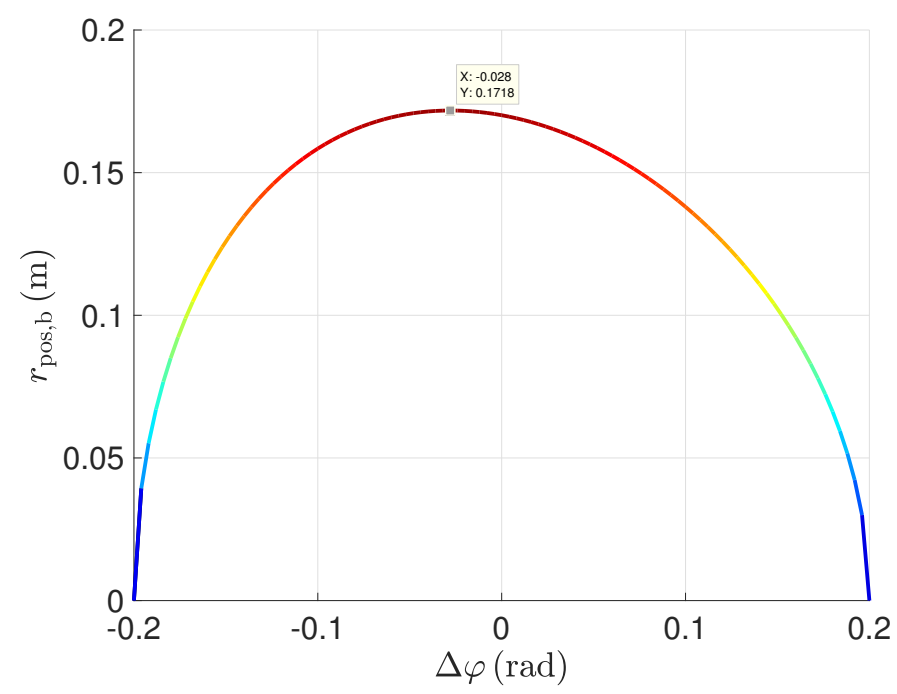

Figure 6: Bundle position radius $\left(t_{l}=1\right)$ versus $\Delta \varphi$.

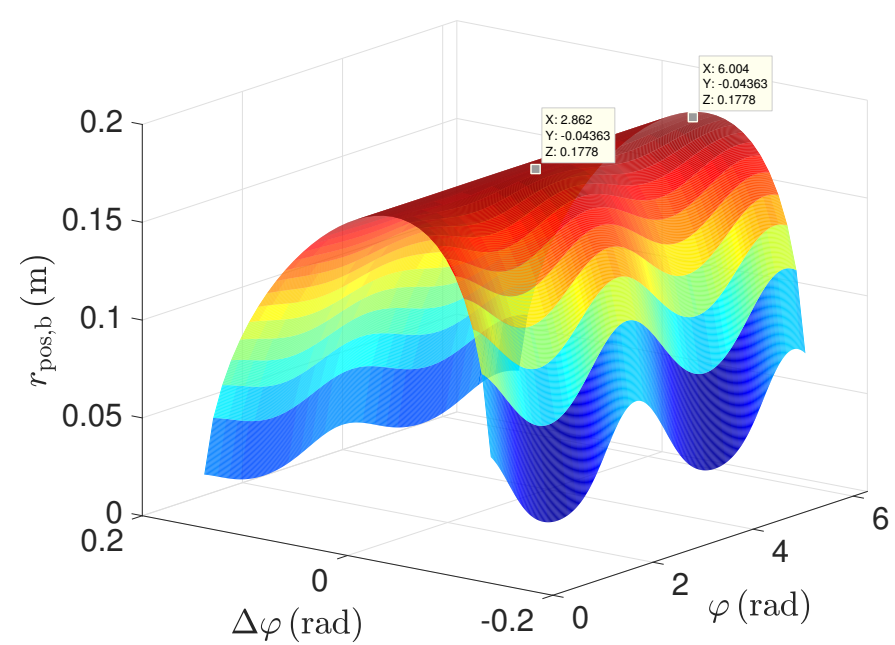

Figure 7: Bundle position radius $\left(t_{l}=2\right)$ versus $\varphi$ and $\Delta \varphi$.

positive) but such a test was taking too long to compute in Matlab for $t_{l}>1$. In order to determine function convexity, a test case for each $t_{l}$ has been selected to plot $r_{\mathrm{pos}, \mathrm{b}}$. The geometric parameters for each case appear on Table 1 .

The only function that is unquestionably convex is that of $t_{l}=1$, as seen on Fig. 6. The function for $t_{l}=2$ is harder to determine (Fig. 7), but all minima 


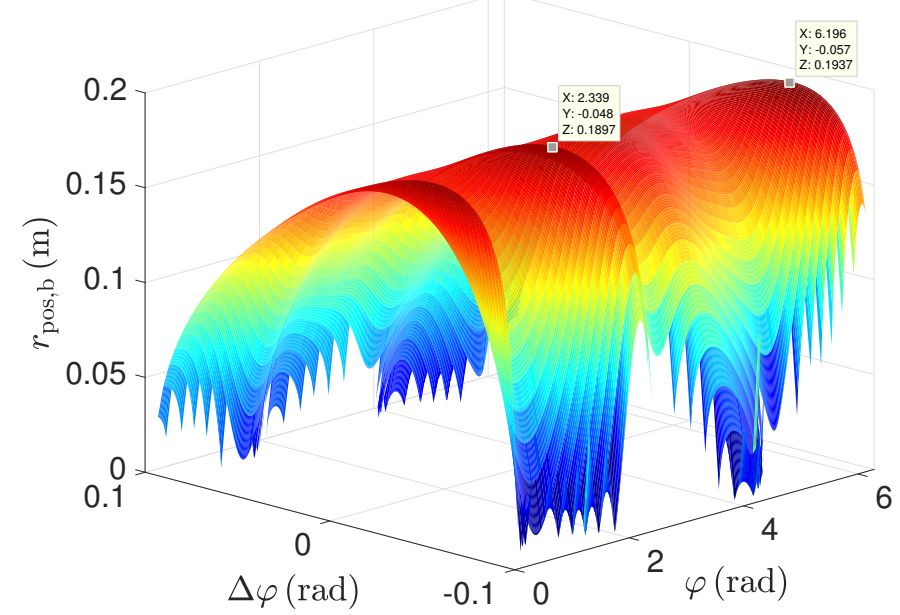

Figure 8: Bundle position radius $\left(t_{l}=3\right)$ versus $\varphi$ and $\Delta \varphi$.

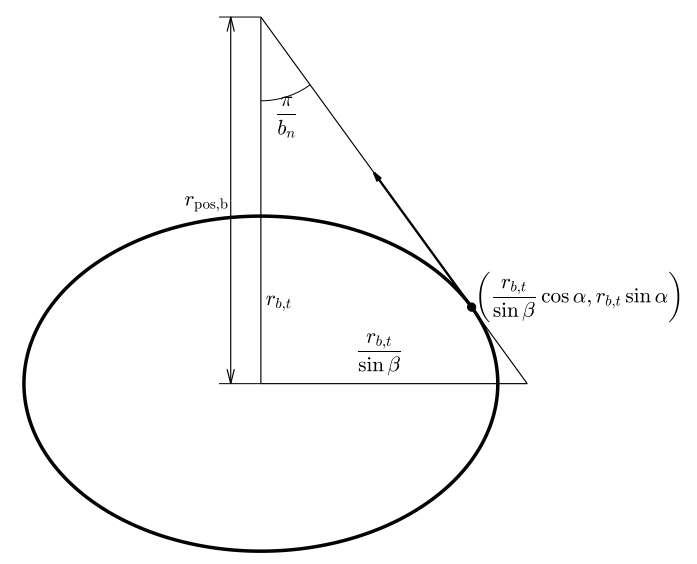

Figure 9: 2D bundle section simplification.

found are global minima, as the function is periodic with period $2 \pi / p r_{2}$ in $\varphi$. Lastly, the function for $t_{l}=3$ is simply not convex, as the global minimum is surrounded by local minima and is difficult to reach (Fig. 8). 


\subsubsection{Two dimensional method}

The two-dimensional method assumes that the sub-bundle sections are created by cuts of the big bundle's normal plane with cylinders of radius $r_{b, t}=$ $r_{b}+t_{\text {ins }} / 2$ and incident angle $\beta$, as shown in Fig. 9 Each bundle layer is made up of identical, regularly spaced $b_{n}$ ellipses with their small axis in the radial direction. Though $\beta$ is used elsewhere for the main cable angle, in this case it is bundle specific. It can be calculated from the scalar product of the central bundle trajectory and the considered bundle's: $\hat{\mathbf{t}}_{n} \cdot \hat{\mathbf{t}}_{n-1}=\cos (\pi / 2-\beta)$. The semi-minor axis equals $r_{b, t}$ and the semi-major axis equals $r_{b, t} / \sin \beta$. In order for all ellipses to be tangent to one another, each ellipse has to occupy $2 \pi / b_{n}$ rad. The tangent point marked on Fig. 9 has to satisfy three conditions: belong to the ellipse, belong to the diagonal line that diverges $\pi / b_{n} \operatorname{rad}$ from the line that connects to the center of the ellipse and have the ellipse's tangent parallel to this line. The parametric equation of an ellipse with semi-major axis $a$ and semi-minor axis $b$ can be expressed as:

$$
\mathbf{p}_{e}=(a \cos \alpha, b \sin \alpha)
$$

where $\mathbf{p}_{e}$ is the position vector of a generic ellipse and $\alpha$ is the curve parameter. The ellipse tangent, $\overline{\mathbf{t}}_{e}$, can be obtained as the position vector gradient:

$$
\overline{\mathbf{t}}_{e}=(-a \sin \alpha, b \cos \alpha) .
$$

Therefore, $\alpha$ has to satisfy:

$$
\begin{gathered}
\frac{b \cos \alpha}{-a \sin \alpha}=\tan \left(\pi-\left(\frac{\pi}{2}-\frac{\pi}{b_{n}}\right)\right), \\
\tan \alpha=\tan \frac{\pi}{b_{n}} \sin \beta .
\end{gathered}
$$

For the ellipse point to be in the diagonal line, this last condition has to be 


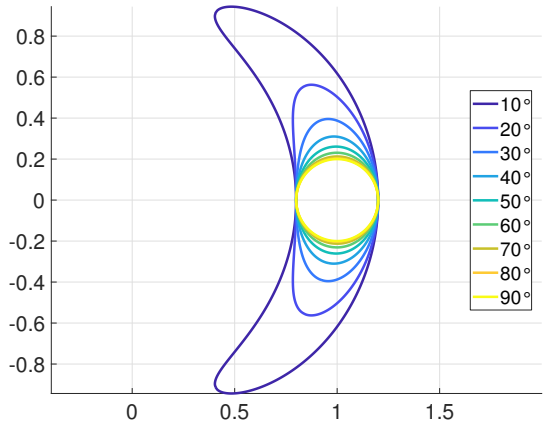

(a)

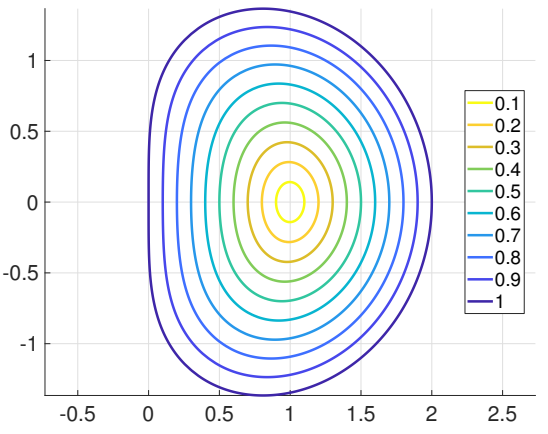

(b)

Figure 10: Real cross sections of bundles depending on (a) helix angle $\beta$ (at ratio 0.2) (b) External and position radius ratio $r_{b, t} / r_{\text {pos,b }}\left(\right.$ at $\left.\beta 45^{\circ}\right)$.

met:

$$
\begin{gathered}
\tan \frac{\pi}{b_{n}}=\frac{\frac{r_{b, t}}{\sin \beta} \cos \alpha}{r_{\mathrm{pos}, \mathrm{b}}-r_{b, t} \sin \alpha}, \\
r_{\mathrm{pos}, \mathrm{b}}=r_{b, t} \sqrt{1+\frac{1}{\tan ^{2} \frac{\pi}{b_{n}} \sin ^{2} \beta}} .
\end{gathered}
$$

If the input parameter is $\lambda$ instead of $\beta$, the expression of $r_{\mathrm{pos}, \mathrm{b}}$ becomes:

$$
r_{\mathrm{pos}, \mathrm{b}}=\frac{r_{b, t}}{\sqrt{\left(1+\frac{r_{b, t}^{2}}{\lambda^{2}}\right) \sin ^{2} \frac{\pi}{b_{n}}-\frac{r_{b, t}^{2}}{\lambda^{2}}}}
$$

The resulting expressions are much simpler than the 3D optimization process, but they become inaccurate as $\beta$ decreases and the ratio $r_{b, t} / r_{\mathrm{pos}, \mathrm{b}}$ increases, as shown on Fig 10. The cuts were generated with the position vector for helices of $t_{l}=2, \mathbf{r}_{2}(\varphi, \alpha)$, where $\varphi$ is the parameter that sweeps the bundle's trajectory while $\alpha$ is the parameter that sweeps the bundle's normal angle. The combination of $\varphi$ and $\alpha$ when they are not bound generates the external bundle surface. The cut with the plane $x=x_{0}$ provides the relation between $\varphi$ and $\alpha$ that describes the parametric curve of the bundle surface contained in the 
Table 2: Calculated $r_{\mathrm{pos}, \mathrm{b}}$ for the 2, 4, 4 case

\begin{tabular}{c|ccc}
$\mathrm{a}$ & $r_{\mathrm{pos}, \mathrm{b}, 1}(\mu \mathrm{m})$ & $r_{\mathrm{pos}, \mathrm{b}, 2}(\mu \mathrm{m})$ & $r_{\mathrm{pos}, \mathrm{b}, 3}(\mu \mathrm{m})$ \\
\hline 2D method & 728.1 & 289.9 & 100.0 \\
3D method & 731.6 & 289.6 & 100.0 \\
absolute difference & 3.476 & 0.6808 & 0 \\
relative difference & $0.457 \%$ & $0.235 \%$ & $0 \%$
\end{tabular}

Table 3: Calculated $r_{\text {pos,b }}$ for the 4, 4, 2 case

\begin{tabular}{c|ccc}
$\mathrm{a}$ & $r_{\mathrm{pos}, \mathrm{b}, 1}(\mu \mathrm{m})$ & $r_{\mathrm{pos}, \mathrm{b}, 2}(\mu \mathrm{m})$ & $r_{\mathrm{pos}, \mathrm{b}, 3}(\mu \mathrm{m})$ \\
\hline 2D method & 602.3 & 355.0 & 142.3 \\
3D method & 602.3 & 359.9 & 142.4 \\
absolute difference & 0 & 4.958 & 0.0824 \\
relative difference & $0 \%$ & $1.38 \%$ & $0.0579 \%$
\end{tabular}

plane:

$$
\begin{aligned}
x_{0} & =\lambda \varphi+r_{b} \cos \beta \sin \left(p r_{2} \varphi+\alpha\right) . \\
\alpha & =\arcsin \left(\frac{x_{0}-\lambda \varphi}{r_{b} \cos \beta}\right)-p r_{2} \varphi .
\end{aligned}
$$

For the solution to be real, $\varphi$ must be bound between $\left(x_{0}-r_{b} \cos \beta\right) / \lambda$ and $\left(x_{0}+r_{b} \cos \beta\right) / \lambda$.

\subsection{Method comparison for the simulations at hand}

The minimum position radii were calculated for each bundle level for both simulation cases, $2 \times 4 \times 4$ and $4 \times 4 \times 2$, assuming zero insulation. The results are shown in Tables 2 and 3 respectively. The maximum relative and absolute difference found is $1.4 \%$ and $5 \mu \mathrm{m}$, significant enough for problems that require zero tolerance, but adequate for magnetic field calculations where the separation between strands is big enough. For the main simulations, $t_{\text {ins }}=30 \mu \mathrm{m}$, so $r_{\mathrm{pos}, \mathrm{b}, 2}$ from this example would increase around $22 \mu \mathrm{m}$ using (27), completely avoiding collision between bundles. 


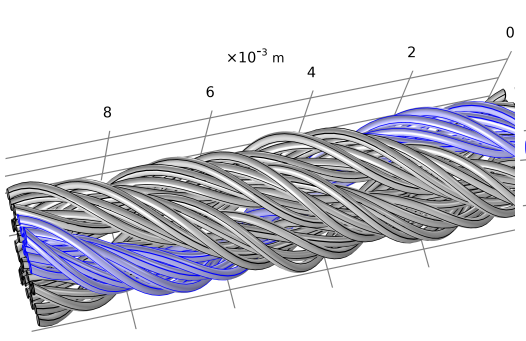

(a)

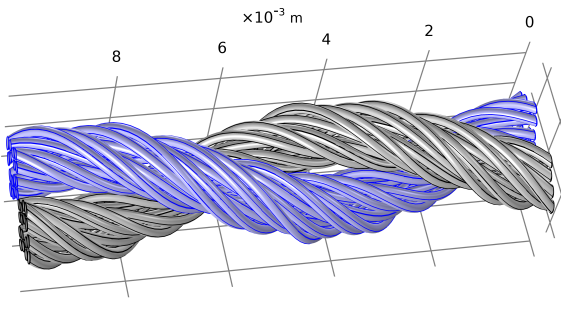

(b)

Figure 11: Geometry of the two simulated cables.

Since the 3D method was implemented for testing purposes and it is more accurate than the 2D method, it was used for the simulations in this paper. Nevertheless, the $2 \mathrm{D}$ method is nearly as accurate and much simpler to implement, so future simulations are encouraged to use this method.

\section{Simulation Model}

The previous mathematical description is used to simulate two different cables with identical number of strands, i.e. $n_{s}=32$, of diameter $\phi_{s}=0.2 \mathrm{~mm}$. Cables are arranged according to the bundling sequence $2 \times 4 \times 4$ and $4 \times 4 \times 2$. The first sequence corresponds to a cable of 4 external bundles composed of 4 bundles of a twisted pair of strands. The second sequence is a twisted pair of bundles whose internal structure consists of 4 bundles of 4 strands. An image of the simulated cables is shown in Fig. 11, where one of the external bundles is highlighted for the sake of clarity.

As it was commented in Section 2, the commercial FEA software COMSOL is used. This software runs in a computer with a i7-3820 CPU $(3.6 \mathrm{GHz}$ clock rate) and $64 \mathrm{~GB}$ of RAM memory. In order to properly capture both skin and proximity effects, which require fine meshing in the radial direction of strands whereas the mesh can be coarser in the longitudinal direction, a meshing strategy based on boundary layers is used. The strands' outer circumference is divided into 20 elements and their length is divided into 200 elements, converting the resulting rectangles into triangles. From the exterior surface, 3 boundary layers are generated inwards to better capture skin effects. The rest 


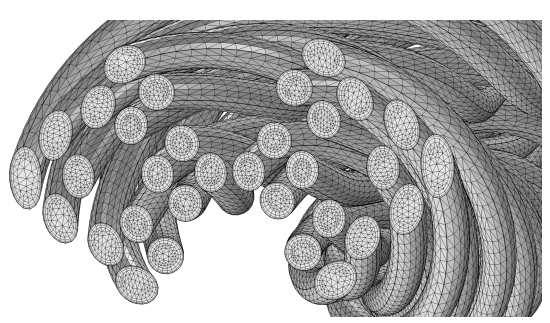

(a)

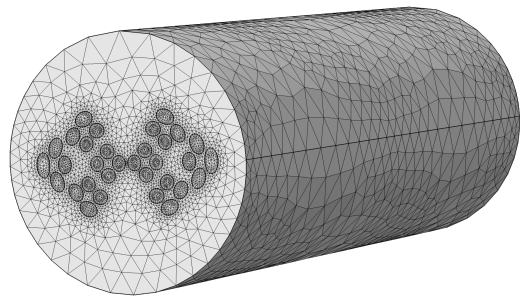

(b)

Figure 12: Mesh of the strands and air.

of the strands' interior as well as the outside air are meshed by free tetrahedra. The total number of elements are 1553317 for the $2 \times 4 \times 4$ cable and 1444346 for the $4 \times 4 \times 2$ cable. Figure 12 shows the mesh of the $4 \times 4 \times 2$ cable with the air hidden in (a) and shown in (b). The simulation of both configurations and all frequencies takes approximately $6 \mathrm{~h} 30 \mathrm{~m}$ to complete and uses $58 \mathrm{~GB}$ of RAM. The 2D simulation used for comparison purposes also has boundary layer meshing, resulting in 115324 triangle elements and taking about $3 \mathrm{~m} 40 \mathrm{~s}$ in total.

The objective of simulation is to obtain the ac resistance of cables and also to check if the proposed 3D description is advantageous with respect to $2 \mathrm{D}$ models, where the transposition of strands is not captured. The transposition should be reflected in an improvement of the equivalence among strands. Ac resistance of cables is the consequence of ac losses due to two purely ac effects: skin and proximity [29]. These effects are simulated by applying different boundary conditions, which correspond to the different physical origin of skin and proximity losses. Skin effect is simulated by applying an ac voltage difference between the wire terminals. Proximity effect refers to losses in a conductive material when it is under an external alternating magnetic field. Therefore, the applied boundary condition consists of an ac magnetic field perpendicular to the cable. Moreover, it is also assumed that the simulated cable is in the air and isolated from other elements. Voltage and field ac dependency is considered sinusoidal.

Loss dissipated in copper strands can be obtained by integrating the Joule's 
losses, which using the microscopic Ohm's law, $\mathbf{J}=\sigma_{C u} \cdot \mathbf{E}$, are expressed as:

$$
P_{s}=\frac{1}{2} \int_{\Omega_{s}} \frac{\mathbf{J} \cdot \mathbf{J}^{*}}{\sigma_{C u}} d v
$$

where $\mathbf{J}$ is the current density in the strand, $\mathbf{J}^{*}$ its conjugated magnitude, and $\Omega_{s}$ is the strand domain.

Total losses of cable corresponds to the sum of losses of all strands, $P_{c}=$ $\sum_{n_{s}} P_{s}$. It is also interesting to connect losses with resistances. For this purpose, total current in the cable is required. As occurs with losses, the total current in the cable is the sum of currents in individual strand, i.e.:

$$
I=\sum_{n_{s}} I_{s}=\sum_{n_{s}} \int_{S_{s}} \mathbf{J} \cdot \hat{\mathbf{n}} \cdot d S
$$

where $S_{s}$ is the cross section of the strand. The normal vector of a strand corresponding to a specific twisting level can be obtained from (6).

In order to compare the resistance of different arrangements, it is also convenient to obtain the power or the resistance per unit of length. The length of a strand $l_{s}$ can be calculated as follows:

$$
l_{s}=\frac{1}{\pi r_{s}^{2}} \int_{\Omega_{s}} d v
$$

\section{Simulation Results}

Fig. 13 shows the simulation results of current density for the two considered arrangements: Fig. 13(a) and Fig. 13(c) for the $2 \times 4 \times 4$ cable, and Fig. 13(b) and Fig. 13(d) for the $4 \times 4 \times 2$ cable. The skin effect corresponds to the upper figures and the proximity effect results to the lower figures. The excitation frequency has been set to $1 \mathrm{MHz}$. At this frequency, a trade off between making evident the ac losses and meshing requirements is achieved. Regarding skin effect and, in view of the scale of the color legend, it could be concluded that current is spread among strands. Regarding proximity effect, it is also observed that twisting leads to similar induced currents in all strands. 


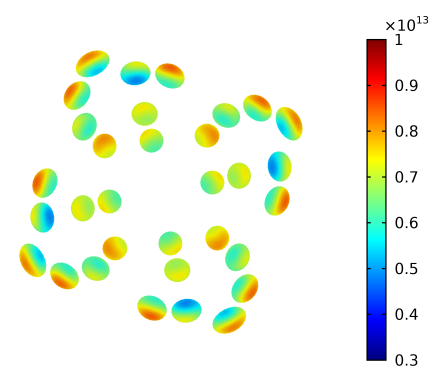

(a)

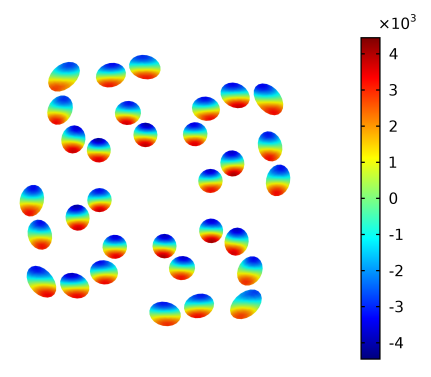

(c)

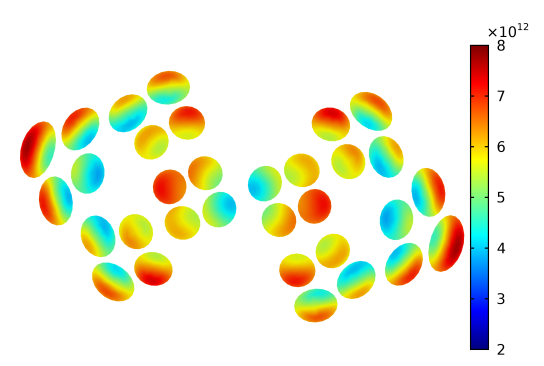

(b)

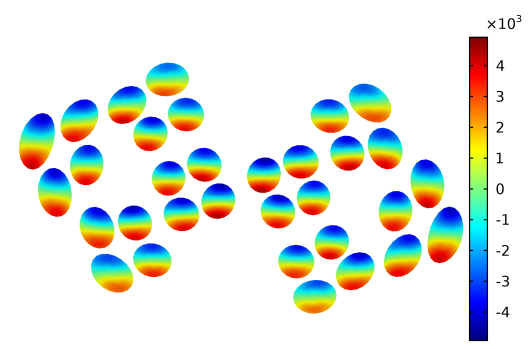

(d)

Figure 13: Current density at $1 \mathrm{MHz}$ for the two considered cable arrangements an ac loss effects. (a) Case $2 \times 4 \times 4$, skin effect. (b) Case $4 \times 4 \times 2$, skin effect. (c) Case $2 \times 4 \times 4$, proximity effect. (d) Case $4 \times 4 \times 2$, proximity effect.

The effectiveness of the modeled cable structures is also shown when acloss results of $2 \mathrm{D}$ and $3 \mathrm{D}$ simulations are compared. The $2 \mathrm{D}$ simulated case corresponds to a cable with identical number of strands and similar arrangement. The frequency of simulations is also set at $1 \mathrm{MHz}$. Results are presented in Fig. 14(a) for the case of the skin effects and Fig. 14(b) for the case of proximity effect. As it is shown, in contrast with external strands, inner strands hardly carry current. This uneven current distribution causes the overestimation of the cable losses and resistance. Moreover, the effect of the non-twisting in the 2D simulation generates induced current whose round-trip path involves several strands, which does not occur when strands are twisted because, in this case, each strand carries an individual induced current, as it is shown in Fig. 13.(b) 


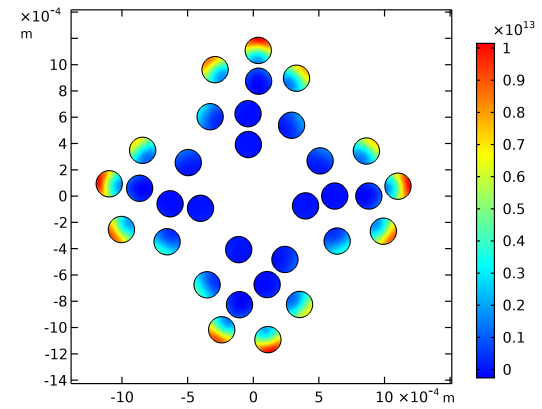

(a)

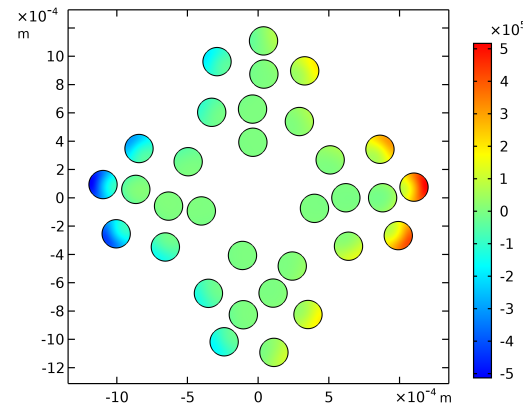

(b)

Figure 14: Current density at $1 \mathrm{MHz}$ for a 2D finite element simulation. (a) Skin effect. (b) Proximity effect.

and Fig. 13(c). Considering that proximity losses are proportional to the square of induced current density, and considering the results obtained in Fig. 13 and Fig. 14, this effect is also reflected in the overestimation of proximity losses. These results point the interest on having proper 3D models.

Qualitative results are interesting for examining the simulation results at a glance. Moreover, quantitative results of the conduction resistance per strand length are also presented. Fig. 15 shows the skin effect resistance for the different modeled cases, relative to the low frequency (near DC) result. In this figure, the maximum and minimum resistances of strands are also compared, which is a measure of its equivalence. The results show that for the $2 \times 4 \times 4$ case the difference between the maximum and minimum resistance values of strands is $8 \%$, whereas this value is about $25 \%$ for the $4 \times 4 \times 2$ case and the 2D simulation. The length difference between the shortest and longest strands is $2.5 \%$, which can further separate the absolute resistance values in the $3 \mathrm{D}$ cases. The preceding results points that different arrangements could lead to different ac resistances and difference equivalence of strands. This fact shows the practical interest of having a proper model for describing complex litz-wire cables. 


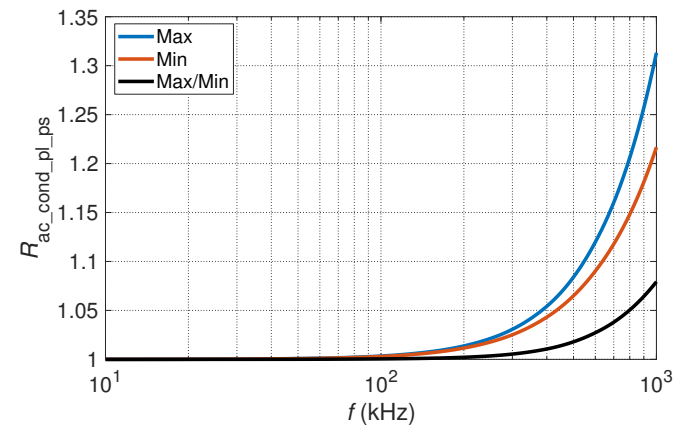

(a)

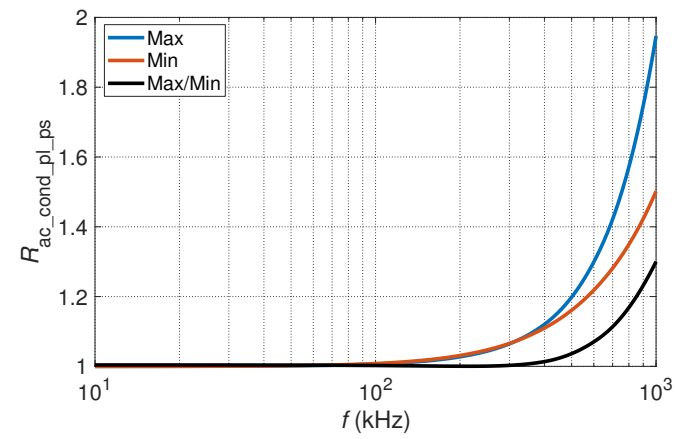

(b)

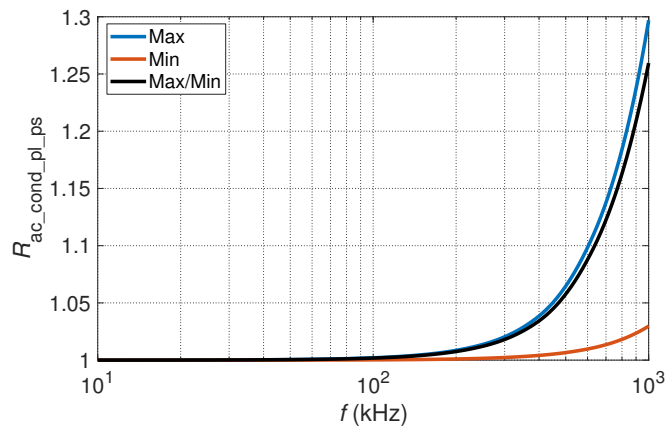

(c)

Figure 15: Comparison of skin effect resistance of strands for different cases. (a) Case $2 \times 4 \times 4$. (b) Case $4 \times 4 \times 2$. (c) Case $2 \mathrm{D}$

\section{Conclusion}

In this work, a geometrical description of rope wires for electrical applications (also called litz wires) is presented. The description is mainly oriented to 
3D finite element simulations and it is based on description of helix or superhelix curves in the Frenet-Serret frame. This description accounts for the complex bundle structure of litz wires, and also considers some peculiarities of these cables with respect to wire rope, as the required insulation between individual strands. In order to denote properly a bundle belonging to a multi-bundle structure, a nomenclature is also proposed. A vector is associated to each bundle whose number of elements and the value of each element correspond with the number of bundling level and the layer number of the bundle. Additionally, in order to avoid collisions between conductors, some methods are also analyzed, learning that despite the inaccuracy of $2 \mathrm{D}$ methods, they can still be recommended for litz wire geometries due to their strand insulation and relatively high pitch.

The developed model is tested by means of 3D finite element simulations with special emphasis in the study of ac-field effects on Joule's losses of cables. Moreover 2D simulations are also carried out for comparison purposes. The model correctness is proven by confirming the improvement of the equivalence among strands achieved with some configurations. It is also shown that twisting cancels the proximity effect at bundle level.

The proposed description can be consider of practical interest for studying the performance of litz wires, in terms of ac resistance, for different arrangements or pitches.

\section{Acknowledgment}

This work was partly supported by the Spanish MINECO under Project TEC2016-78358-R, by the Spanish MICINN and AEI under Project RTC-20175965-6, co-funded by EU through FEDER program, by the BSH Home Appliances Group and by the Gobierno de Aragón-FSE 2014-20 under Grant IIU $/ 2023 / 2017$. 


\section{References}

[1] Z. Zhang, H. Pang, A. Georgiadis, C. Cecati, Wireless power transfer: An overview 66 (2019) 1044-1058.

[2] J. Acero, R. Alonso, J. M. Burdio, L. A. Barragan, D. Puyal, Frequencydependent resistance in litz-wire planar windings for domestic induction heating appliances 21 (4) (2006) 856-866.

[3] D. I. Zaikin, S. Jonasen, S. L. Mikkelsen, An air-gap shape optimization for fringing field eddy current loss reductions in power magnetics, IEEE Transactions on Power Electronics 34 (5) (2019) 4079-4086.

[4] J. Acero, C. Carretero, I. Lope, R. Alonso, J. Burdío, Analytical solution of the induced currents in multilayer cylindrical conductors under external electromagnetic sources, Applied Mathematical Modelling (2016).

URL http://dx.doi.org/10.1016/j .apm.2016.07.031

[5] W. Hurley, W. Wölfle, Transformers and Inductors for Power Electronics. Theory, Design and Applications, John Wiley \& Sons, United Kingdom, 2013.

[6] J. Schutz, J. Roudet, A. Schellmanns, Modeling litz wire windings, in: Thirty-Second IAS Annual Meeting, IAS '97., Conference Record of the 1997 IEEE Industry Applications Conference, 1997., 1997, pp. 1190-1195.

[7] J. Gyselinck, P. Dular, Frequency-domain homogenization of bundles of wires in 2-d magnetodynamic fe calculations, IEEE Transactions on Magnetics 41 (5) (2005) 1416-1419.

[8] B. A. Reese, R. Joseph, C. R. Sullivan, Improved litz-wire designs for the mhz range, in: 2018 IEEE 19th Workshop on Control and Modeling for Power Electronics (COMPEL), 2018.

[9] R. W. M. Kazimierczuk, Winding resistance and power loss of inductors with litz and solid-round wires, IEEE Transactions on Industry Applications 54 (4) (2018) 3548-3557. 
[10] C. Carretero, J. Acero, R. Alonso, Tm-te decomposition of power losses in multi-stranded litz-wires used in electronic devices, Progress In Electromagnetics Research, PIER 123 (1) (2012) 83-103.

[11] G. Cerri, S. Kovyryalov, V. Primiani, Modelling of a litz-wire planar winding, Science, Measurement \& Technology, IET 4 (4) (2010) 214-219.

[12] K. Umetani, J. Acero, H. Sarnago, O. Lucia, E. Hiraki, Simple fully analytical copper loss model of litz wire made of strands twisted in multiple levels, in: 2019 IEEE Applied Power Electronics Conference and Exposition (APEC), 2019, pp. 1257-1264.

[13] C. Sullivan, R. Zhang, Simplified design method for litz wire, in: Applied Power Electronics Conference and Exposition (APEC), 2014 Twenty-Ninth Annual IEEE, 2014, pp. 2667-2674.

[14] I. Lope, J. Acero, J. Burdio, C. Carretero, R. Alonso, Design and implementation of pcb inductors with litz-wire structure for conventional-size large-signal domestic induction heating applications, IEEE Transactions on Industry Applications 3 (51) (2015) 2434-2442.

[15] C. R. Sullivan, R. Y. Zhang, Analytical model for effects of twisting on litz-wire losses, in: Proc. IEEE Workshop on Control and Modeling for Power Electronics (COMPEL'14), 2014.

[16] T. Guillod, J. Huber, F. Krismer, J. Kolar, Litz wire losses: Effects of twisting imperfections, in: 2017 IEEE 18th Workshop on Control and Modeling for Power Electronics (COMPEL), 2017.

[17] D. Meeker, An improved continuum skin and proximity effect model for hexagonally packed wires, Journal of Computational and Applied Mathematics 236 (18) (2012) 4635-4644.

[18] R. Scapolan, A. Gagnoud, Y. Terrail, 3-d multistrands inductor modeling: Influence of complex geometrical arrangements, IEEE Transactions on Magnetics 50 (2) (2014) 949-952. 
[19] J. Acero, I. Lope, J. Burdio, C. Carretero, R. Alonso, Loss analysis of multistranded twisted wires by using 3d-fea simulation, in: Proc. IEEE Workshop on Control and Modeling for Power Electronics (COMPEL'14), 2014.

[20] R. Y. Zhang, J. K. White, J. G. Kassakian, Fast simulation of complicated 3-d structures above lossy magnetic media, IEEE Transactions on Magnetics 50 (10) (Oct. 2014).

[21] A. Rosskopf, E. Bar, C. Joffe, C. Bonse, Calculation of power losses in litz wire systems by coupling fem and peec method 31 (9) (2016) 6442-6449.

[22] S. Hiruma, H. Igarashi, Fast 3-d analysis of eddy current in litz wire using integral equation, IEEE Transactions on Magnetics 53 (6) (Jun. 2017).

[23] G. A. Costello, Theory of Wire Rope, Springer, New York, 1990.

[24] X. Wang, X. Meng, J. Wang, Y. Sun, K. Gao, Mathematical modeling and geometric analysis for wire rope strands, Applied Mathematical Modelling 39 (2015) 1019-1032.

[25] P. Zhang, M. Duan, J. Ma, Y. Zhang, A precise mathematical model for geometric modeling of wire rope strands structure, Applied Mathematical Modelling 76 (2019) 151-171.

[26] E. Plumed, J. Acero, I. Lope, C. Carretero, 3D finite element simulation of litz wires with multilevel bundle structure, in: Proc. IECON 2018 44th Annual Conf. of the IEEE Industrial Electronics Society, 2018, pp. 3479-3484. doi:10.1109/IECON.2018.8591341.

[27] L. Makong, A. Kameni, F. Bouillault, P. Masson, 3-d equivalent model to compute the electro-magnetic behavior of twisted multi-filamentary superconductors wires 54 (3) (2018) 1-4.

[28] Mathworks, Find minimum of constrained nonlinear multivariable function - matlab fmincon, Accessed on 29/10/2019.

URL https ://uk.mathworks . com/help/optim/ug/fmincon.html 
[29] J. Lammeraner, M. Stafl, Eddy currents, Iliffe books Ltd. London, 1966. 